\title{
Colonização intradomiciliar de Panstrongylus megistus (Hemiptera, Reduviidae, Triatominae) em São José do Cerrito, SC: primeiro relato
}

\author{
Colonization by Panstrongylus megistus (Hemiptera, Reduviidae, Triatominae) \\ inside homes in São José do Cerrito, SC: first report
}

\author{
Carlos José Raupp Ramos ${ }^{1,2}$, Kaio César Simiano Tavares, \\ Larissa Kaori Oide Komati ${ }^{1}$ e Luiz Claudio Miletti ${ }^{1}$
}

\begin{abstract}
RESUMO
Em resposta a uma denúncia da existência de triatomíneos em uma cabana de pesca as margens do Rio Caveiras no município de São José do CerritoSC, foi feita a investigação na cabana e em outros ecótopos vizinhos ao achado inicial. Foram encontrados 15 exemplares de Panstrongylus megistus sendo que nenhum deles encontrava-se infectado. 0 local do ecótopo é visitado ocasionalmente por capivaras, gambás, tatus e ratos.
\end{abstract}

Palavras-chaves: Panstrongylus megistus. Ecótopo artificial. Santa Catarina. Trypanosoma spp.

\begin{abstract}
:
In response to complaints of the presence of triatomines in a fishing hut on the banks of the Caveiras river, in the municipality of São José do Cerrito, State of Santa Catarina, an investigation was conducted in this hut and in other ecotopes neighboring the initial finding. Fifteen specimens of Panstrongylus megistus were found and none of them were infected. The locality of this ecotope is visited occasionally by capybaras, opossums, armadillos and rats.
\end{abstract}

Key-words: Panstrongylus megistus. Artificial ecotope. Santa Catarina. Trypanosoma spp.

O Panstrongylus megistus (Burmeister, 1835) (Hemiptera, Reduviidae) é um triatomíneo amplamente distribuído no Brasil9, sendo que na região Sul é considerado eminentemente silvestre ${ }^{1}$. O Município de São José do Cerrito, na qual surgiu à denúncia avaliada neste trabalho, encontra-se da microrregião dos Campos de Lages (Figura 1), a 910m de altitude em relação ao nível do mar, faixa considerada dentro dos limites de altitude de encontro para Panstrongylus megistus ${ }^{3}$. Entretanto, segundo Foratini ${ }^{6}$, essa região no Estado de Santa Catarina, por suas características geográficas e ambientais não oferece a princípio condições para a presença de vetores, uma vez que faz parte de uma região intermediária entre o sistema da Serra do Mar e as regiões Noroeste do Rio Grande do Sul e Sudoeste do Paraná, onde ocorre a domiciliação potencial destes triatomíneos.

Embora o Estado de Santa Catarina seja considerado não endêmico para a doença de Chagas, estudos prévios feitos na Iha de Santa Catarina revelaram um índice de infecção natural por Trypanosoma cruzi de 84,5\% e 53,3\% ${ }^{10}$ em Panstrongylus megistus, esses mesmos estudos também indicam algum potencial dessa espécie para invadir e colonizar moradias humanas assim como observado no Estado de São Paulo ${ }^{7}$, Paraná ${ }^{4}$ e em ilhas do Alto Rio Paranás. 0 presente trabalho é o primeiro relato de colonização intradomiciliar de Panstrongylus megistus nesta área do Estado de Santa Catarina.

Em novembro de 2007, um morador da Cidade de LagesSC que possui uma cabana de pesca, feita de costaneira, as margens do Rio Caveiras no Município de São José do Cerrito, vizinho a Lages (Figura 2), notificou a Vigilância Epidemiológica Estadual ( $27^{\text {a }}$ Gerência Regional de Saúde de Lages - $27^{\text {a }}$ GERSA) quanto à presença de insetos semelhantes a barbeiros nessa propriedade, levando um exemplar que foi identificado pelo setor de entomologia da $27^{\mathrm{a}}$ GERSA como sendo um triatomíneo da espécie Panstrongylus megistus. Após a identificação positiva, uma equipe formada por pesquisadores do Centro de Ciências Agroveterinárias da UDESC e da $27^{\mathrm{a}}$ GERSA desenvolveu busca ativa de espécimes e avaliação dos ecótopos naturais próximos à

1.Centro de Ciências Agroveterinárias, Universidade do Estado de Santa Catarina, Lages, SC. 2. Laboratório de Entomologia, $27^{\mathrm{a}}$ Gerência Regional de Saúde de Lages, Divisão de Vetores, Roedores e outros Hospedeiros, Gerência de Zoonoses, Diretoria de Vigilância Epidemiológica - DIVE, Secretaria de Estado da Saúde, Lages, SC.

Endereço para correspondência: Dr. Luiz Claudio Miletti. Curso de Pós-Graduação em Ciência Animal. Av. Luis de Camões 2090, 88520-000 Lages,SC.

Tel: $55492101-9175$

e-mail:miletti@cav.udesc.br

Recebido para publicação em 17/04/2008

Aceito em 02/07/2008 


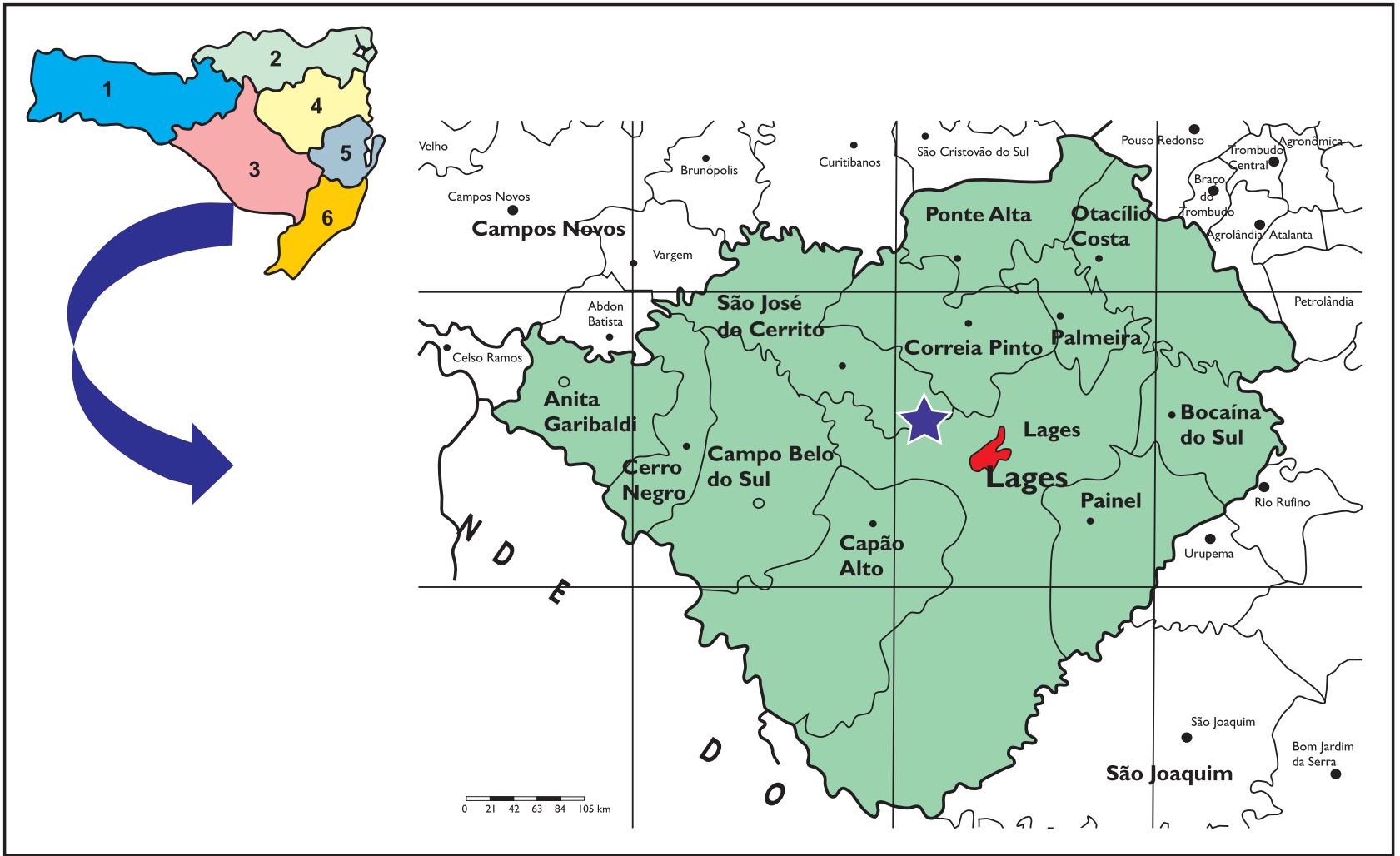

Figura 1 - Posição geográfica do município de São José do Cerrito, onde foram encontrados os exemplares de Panstrongylus megistus, na mesorregião serrana ( $\left.{ }^{\circ} 3\right)$ no mapa do Estado de Santa Catarina. A estrela representa a localização do achado.

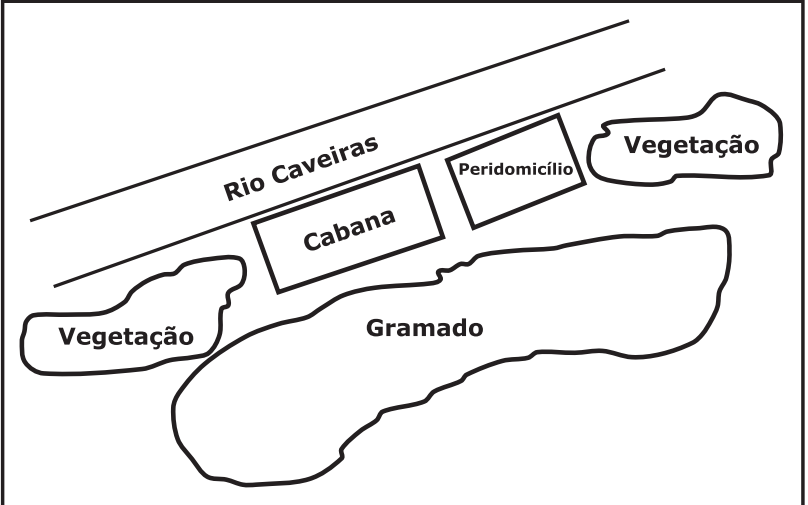

Figura 2 - Croqui do local do foco e arredores.

cabana. Observou-se que a cabana de pesca (Figura 3), apresentava características típicas da zona rural, com estrutura de madeira de Pinus rústicos, sem reboco externo e parte anexa, também de madeira recobertas por costaneira. 0 peridomicílio encontravase desorganizado, mas pouco denso, com acúmulo de materiais diversos (madeira, ferragens, etc). Ainda no peridomicílio, foram observadas várias palmeiras, locais considerados como possíveis habitats dessa espécie de triatomíneo ${ }^{2}$. No intradomicílio, foram encontradas ninfas no armário embutido da cozinha, cuja parede do fundo era a mesma da cabana, que foi colonizado por roedores (não encontrados). Neste mesmo mês, foram coletados 15 triatomíneos da residência, consistindo de 15 ninfas de $1^{\circ}$ a $5^{\circ}$ estádios, identificadas como Panstrongylus megistus pelo Laboratório de Entomologia da $27^{\mathrm{a}}$ GERSA e também pelo Laboratório de Transmissores de Hematozoários do Departamento

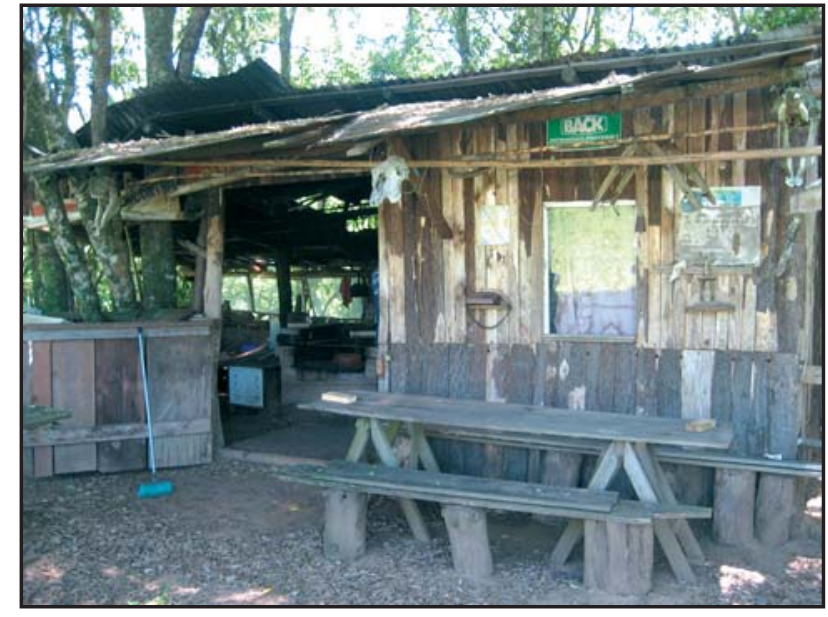

Figura 3 - Cabana de pesca as margens do Rio Caveiras, na qual foram encontrados os exemplares de Panstrongylus megistus.

de Microbiologia e Parasitologia da UFSC. Não foram encontrados na primeira visita triatomíneos adultos nem ovos. No exame de esfregaço das fezes dos triatomíneos, corados com panótico não foram observadas formas flageladas de Trypanosoma sp. Em uma segunda visita feita no mês de março de 2008, encontrou-se novamente um ninho de rato. Foi feita aplicação de desalojante, entretanto nenhum inseto vivo foi observado, sendo encontrado apenas um exemplar morto de um macho adulto, provavelmente pelo inseticida aplicado pelo proprietário. Existe a possibilidade de que os triatomíneos tenham sido carregados para o interior do domicílio nas folhas carregadas pelos roedores. Como a região é habitada por capivaras, gambás, tatus e ratos, novos trabalhos de prospecção serão feitos para avaliar a possibilidade 
de infecção destes hospedeiros na região, que são considerados fauna relacionada com essa espécie de triatomíneo ${ }^{2}$, visando diminuir os riscos de infecções humanas, se Trypanosoma cruzi for encontrado.

\section{AGRADECIMENTOS}

Ao Sr. Elecy Wolff Mota pelo aviso do encontro dos triatomíneos e por possibilitar a busca de outros exemplares em sua propriedade.

\section{REFERÊNCIAS}

1. Aragão MB. Sobre a domiciliação dos triatomíneos. Revista da Sociedade Brasileira de Medicina Tropical 14: 13-27, 1981.

2. Carvalho RU, Galíndez-Girón IG, Jurberg J, Lent H (orgs) Atlas of Chagas' Disease Vectors in the Americas. Fundação Osvaldo Cruz, Rio de Janeiro, volume 2, 1998.

3. Carvalho RU, Galíndez-Girón IG, Jurberg J, Lent H. Atlas of Chagas' Disease Vectors in the Americas. Fundação Osvaldo Cruz, Rio de Janeiro, volume 3, 1999.
4. Falavigna-Guilherme AL, Pavanelli GC, Costa AL, Araújo SM. Prevalence and infections in triatomíneos captured in artificial ecotopes in municipalities under epidemiological surveillance in northwest Paraná, Brazil. Revista Panamericana de Salud Pública 9: 385-392, 2001a.

5. Falavigna-Guilherme AL, Lorosa ES, Costa AL, Pavanelli GC, Araújo SM. Panstrongylus megistus em ecótopos artificiais de ilhas do Alto Rio Paraná. Revista da Sociedade Brasileira de Medicina Tropical 34: 491-494, 2001b.

6. Foratini OP. Biogeografia, origem e distribuição da domiciliação de triatomíneos no Brasil. Revista de Saúde Pública São Paulo 14: 265-299, 1980.

7. Nascimento C, Marassá AM, Curado I, Piazza RMF. Encontro de Panstrongylus megistus em ecótopo artificial: Domiciliação ou mera visitação? Revista da Sociedade Brasileira de Medicina Tropical 30: 333-336, 1997.

8. Schlemper Jr BR, Steindel M, Gargioni R, Farias CJM, Oliveira R, Trianon JAX. Reservatórios e vetores silvestres do Trypanosoma cruzi e suas relações com o domicílio humano na Ilha de Santa Catarina. Arquivos Catarinenses de Medicina 14: 91-96, 1985.

9. Silveira AC, Feitosa V, Borges R. Distribuição de triatomíneos capturados no ambiente domiciliar no período de1975/83 no Brasil. Revista Brasileira de Malária 36: 15-312, 1984.

10. Steindel M, Toma HK, Pinto CJC, Grisard EC, Schlemper Jr BR. Colonização de ecótopos artificiais pelo Panstrongylus megistus na Ilha de Santa Catarina, Florianópolis Santa Catarina, Brasil. Revista do Instituto de Medicina Tropical São Paulo 36: 43-50, 1994. 